\section{EMBRYRIDDLE Aeronautical University}

SCHOLARLY COMMONS
International Journal of Aviation, Aeronautics, and Aerospace

\title{
An Examination of the Relationships between Safety Culture Perceptions and Safety Reporting Behavior among Non-Flight Collegiate Aviation Majors
}

\author{
Daniel Kwasi Adjekum \\ University of North Dakota, kadjekum@yahoo.com \\ Julius Keller \\ Purdue University, keller64@purdue.edu \\ Micah Walala \\ Purdue University, micahwalala@gmail.com \\ Cody Christensen \\ South Dakota State University, cody.christensen@sdstate.edu \\ Randal J. DeMik \\ Lewis University, demikra@lewisu.edu \\ John P. Young \\ Purdue University, jpy@purdue.edu \\ Gary J. Northam

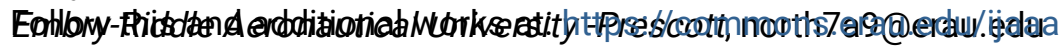 \\ Part of the Bilingual, Multilingual, and Multicultural Education Commons, Community College \\ Leadership Commons, Curriculum and Social Inquiry Commons, Educational Assessment, Evaluation, and \\ Research Commons, Social and Behavioral Sciences Commons, Social and Philosophical Foundations of \\ Education Commons, and the Student Counseling and Personnel Services Commons
}

\section{Scholarly Commons Citation}

Adjekum, D. K., Keller, J., Walala, M., Christensen, C., DeMik, R. J., Young, J. P., \& Northam, G. J. (2016). An Examination of the Relationships between Safety Culture Perceptions and Safety Reporting Behavior among Non-Flight Collegiate Aviation Majors. International Journal of Aviation, Aeronautics, and Aerospace, 3(3). https://doi.org/10.15394/ijaaa.2016.1134

This Article is brought to you for free and open access by the Journals at Scholarly Commons. It has been accepted for inclusion in International Journal of Aviation, Aeronautics, and Aerospace by an authorized administrator of Scholarly Commons. For more information, please contact commons@erau.edu. 


\section{An Examination of the Relationships between Safety Culture Perceptions and Safety Reporting Behavior among Non-Flight Collegiate Aviation Majors}

\section{Cover Page Footnote}

The researchers are grateful to the leadership of all the participating universities for the assistance in undertaking this research. 
Some collegiate aviation programs in the United States have adopted proactive safety initiatives such as the voluntary Safety Management System (SMS) program which is strongly advocated by the Federal Aviation Administration (FAA). The International Civil Aviation Organization (ICAO) defines SMS as an organized approach to managing safety, including the necessary organizational structures, accountabilities, policies, and procedures (ICAO, 2013). The FAA defines SMS as a formal, top-down, organization-wide approach to managing safety risks, and enhancing the effectiveness of safety risk controls (FAA, 2015). An SMS consists of four main components: Safety Policy and Objectives, Safety Risk Management, Safety Assurance, and Safety Promotion. These four main components comprise twelve sub-components reflected in both the ICAO and FAA SMS manuals (FAA, 2015; ICAO, 2013).

Although all components of an SMS are important, safety risk management and safety assurance actively seek hazards and reduce risks, while safety policy and promotion have supporting features (FAA, 2012; ICAO, 2009). Successful implementation of such safety programs assist in reducing safety occurrences and ensure the flight program exceeds minimum regulatory compliance (FAA, 2012; FAA, 2015). Collegiate programs that participate in the voluntary SMS programs are not under strict implementation and compliance when compared to Part 121 and some Part 135 operators, under the new Part 5 SMS rule.

The Part 5 SMS rule requires operators authorized to conduct operations under Part 121 to develop and implement a Safety Management System. The rule creates the general framework for an SMS that a Part 121 air carrier may adapt to fit the needs of its operation. It also modifies title 14 CFR Part 119 to specify applicability and implementation of the new SMS framework in part 5 for aircraft operators certificated under Part 121 (FAA, 2015). Even though these collegiate programs do not fall under the Part 5 rule, they need to meet some performance requirements of SMS such as a proactive safety management processes and effective safety reporting systems in order to maintain their active conformant status in the SMS voluntary program for training organizations (FAA, 2015).

Extant research suggests a link between the safety culture perceptions of the flight operational personnel (e.g. flight students and flight instructors) and behaviors such as the reporting of safety occurrences (Adjekum, 2014; Adjekum et. al, 2015; Dillman, Voges \& Robertson, 2010; Drinkwater \& Molesworth, 2010; Hunter, 2006; Molesworth \& Chang, 2009). Most of these literatures have focused on the impact of SMS implementation on the safety culture perceptions and behavior of flight operational personnel and there have been suggestions that SMS may positively affect the safety culture and increase desired safety behaviors of 
these personnel in collegiate aviation programs (Adjekum, 2014; Adjekum et al., 2015).

There seems to be paucity of literature on the safety culture perceptions of non-flight majors such as air traffic control (ATC) students, aviation management (AVMgt), and unmanned aircraft systems (UAS) students and its impact on a successful implementation of SMS in collegiate aviation programs, especially when these students often share the operational environment with traditional flight students. Effective SMS programs should target and include all personnel in an organization. The non- inclusivity of some students in the broad safety policy, promotion, and risk management programs, required under SMS protocol, may render these groups of students as weak links (Reason, 2008; Stolzer, Halford \& Goglia, 2011). A study which examines the perceptions of non-flight collegiate aviation majors and the effect on safety reporting behavior may improve SMS implementation and continuing processes.

This paper is the final of a broad-based study on collegiate aviation safety culture assessment conducted in 2015. The purpose of this paper is to examine the relationships between safety culture perceptions of non-flight collegiate aviation majors such as ATC, AVMgt, and UAS students regarding the propensity to selfreport safety events. The study also analyzed the effect of age on safety culture variables and the inclination to report safety occurrences. Researchers modified and distributed safety culture scales from the Collegiate Aviation Perception of Safety Culture Assessment Scale (CAPSCAS), which is a validated survey instrument with a reported reliability range of ( $\alpha=.86$ to .92) (Adjekum, 2014; Adjekum et al., 2015). Exploratory Factor Analysis (EFA), Confirmatory Factor Analysis (CFA), and Structural Equation Modeling (SEM) techniques were used in the analyses of the modified survey instrument.

\section{Literature Review}

Desired safety culture perceptions of all the stakeholders within the operational environment have become essential due to the moderating influence on individual safety behaviors (Cooper, 2000; Dillman, Voges \& Robertson, 2010; Freiwald, Lenz-Anderson \& Baker, 2013). Safety managers should identify predictive relationships between safety culture perceptions of personnel and their safety behavior (FAA, 2012; Hudson, 2001). Studies suggested that safety culture perceptions of personnel could have an influence on safety behavior in terms of compliance, participation, and frequency of self-reporting hazards (DeJoy, Schaffer, Wilson, Griffin \& Neal, 2000; Mearns, Whitaker, \& Flin, 2003; Vandenberg \& Butts, 2004; Zohar \& Luria, 2004). The measurement scales used to 
assess safety culture perceptions in this study were: safety values, safety fundamentals, response and feedback, reporting system, and aviation department safety record (Adjekum et al., 2015.). These scales are explained further in the following section.

Beyond an organization's physical environment, an individual's perception of the organization's commitment to safety may also influence attitudes and actions in promoting a good safety culture (Cooper, 2000). It may be insightful to examine how respondents perceive organizational compliance with safety-related regulations and how these perceptions affect safety-reporting frequency. While examining the relationship between perceived compliance and reporting frequency, it may also be important to determine the mediating role of personnel perceptions on safety values. Safety values are attitudes and values of program leadership regarding safety, expressed in words and actions (Adjekum et al., 2015). Personnel may lose confidence in safety processes if leadership is perceived as not proactive, does not provide the necessary resources, and does not participate in safety initiatives. The propensity of personnel to self-report safety issues and identify hazards may provide the necessary input for safety managers. Data may be used to apply safety risk mitigation, control strategies, continuous improvement efforts, and bolster safety fundamentals of the program.

According to Adjekum et al. (2015), another essential variable that influences collegiate aviation flight students and instructors was the quality of the safety reporting system. The researchers posited that the perceptions of how anonymous or confidential a reporting system was, invariably influenced the reporting behavior of respondents. Additionally, a reporting system that is easy to access and to complete a report may increase the desire to use it. Furthermore, researchers observed a significant linear relationship, between respondent age group, and the frequency of safety reporting.

The researchers (Adjekum et al., 2015) suggested reasons for the trend of less reporting by older respondents could be that older respondents were more comfortable with operational risks. It may also be older respondents, with relatively extensive operational experience and knowledge may demonstrate cautious proactive safety attitudes, which may reduce safety occurrences and lower safety reporting. The authors also suggested further research should investigate the mediating role of perceptions about the quality of the reporting system between age and reporting frequency (Adjekum et al., 2015).

Positive safety culture perceptions may increase desired behaviors such as reporting. Negative safety culture perceptions of personnel could negatively 
influence safety behavior resulting in incidents and accidents (Chen, 2014; Hunter, 2006; Dillman, Voges, \& Robertson, 2010). According to Cooper (2000), incidents and accidents may lead to the loss of lives, damage to equipment, tarnished reputations, and loss of confidence by customers. Perceptions of safety culture variables and demographics may affect safety behavior, particularly for flight students and instructors. It is intuitive to further examine the link between safety culture perceptions, age, and reporting behavior among collegiate aviation students other than flight personnel.

\section{Research Purpose and Questions}

The purpose of the study was to assess the relationship between the safety culture perception, demographic variables, and the safety reporting behavior of respondents (collegiate ATC, Aviation Management, and UAS students) at five collegiate aviation programs, which are located in the Midwestern and Southwestern parts of the United States. All of the collegiate aviation institutions have fully accredited 14 Code of Federal Regulations (CFR) Part 141 aviation programs, and are at various implementation stages of the voluntary SMS program. The target population in the current study was undergraduate and graduate students enrolled in ATC, UAS, and Aviation Management courses. The researchers in this study addressed the following research questions:

1. What is the validity of the measurement models for the safety culture scales: Safety Values, Safety Fundamentals, Response and Feedback, Reporting System, and Aviation Department Safety Record within a collegiate aviation program?

2. What is the magnitude of the overall perceptions of respondents on the safety culture scales: Safety Values, Safety Fundamentals, Response and Feedback, Reporting System, and Aviation Department Safety Record?

3. What are the strengths of the relationships between respondent perceptions on the safety culture scales: Safety Values, Safety Fundamentals, Response and Feedback, Reporting System, Aviation Department Safety Record, demographic variables, and frequency of self- reporting safety issues by respondents?

4. What is the relationship between Age variable and frequency of selfreporting safety issues when mediated by reporting system?

5. What is the relationship between Safety Fundamentals and frequency of self-reporting of safety issues when mediated by the Safety Values?

The following hypotheses were generated for questions four and five based on the review of literature and the research questions: 
$\mathbf{H}_{1}$ : There is a relationship between Age variable and frequency of selfreporting safety issues.

$\mathbf{H}_{2}$ : There is a relationship between Age variable and frequency of selfreporting safety issues when mediated by Reporting System.

H3: There is a relationship between the variables Safety Fundamentals and frequency of self- reporting safety issues.

H4: There is a relationship between the variables Safety Fundamentals and frequency of self- reporting safety issues when mediated by Safety Values.

\section{Method}

A cross-sectional quantitative study was used to assess the relationship between the safety culture perception, demographic variables, and the safety reporting behavior of respondents (collegiate ATC, Aviation Management, and UAS students) at five collegiate aviation programs. The cross-sectional study design allowed the researchers to compare different study variables at the same time even though the design has a limitation in adducing cause-and-effect relationships (Creswell, 2009). The study was grounded in Cooper's safety culture theory that the perceptions of respondents on organizational safety culture influenced their safety behavior (Cooper, 2000).

\section{Research Instrument}

Researchers of the current study adopted items for this study from the Collegiate Aviation Perception of Safety Culture Assessment Survey (CAPSCAS). The CAPSCAS is a survey instrument for assessing safety culture and SMS within collegiate aviation. The modified version of the CAPSCAS used for this study had five scales, each having multiple survey items. The five scales are Safety Values, Safety Fundamentals, Reporting System, Response Feedback, and Aviation Department Safety Record.

The Safety Value (SV) scale refers to the attitudes and values regarding safety, expressed in words and actions by collegiate aviation leadership. Safety Fundamentals (SF) are concerned with how an aviation organization is set up in relation to compliance with regulated aspects of safety such as training requirements, Standard Operating Procedures (SOPs) and other technical manuals. The Reporting System (RS) refers to the accessibility, familiarity, and actual use of operational personnel and end users.

Response and Feedback (RF) entails the timeliness and appropriateness of management responses to reported safety information, and dissemination of safety 
information to operational personnel. It is also a measure of the quality of feedback on safety reports by the organization's leadership to relevant personnel. The Aviation Department Safety Record (ADSR) pertains to respondent perceptions of the overall safety record and regulatory compliance capabilities of the organization. It is also an effective gauge of the probability of safety and regulatory violations.

The Safety Behavior (SB) sub-scale is the dependent variable, defined as the frequency of respondent's voluntary self-reporting of hazards and other observed safety issues. An assumption for this study was self-reporting of safety issues by respondents were driven by perceptions of the safety culture in the operations of the aviation department (Adjekum, 2014). The CAPSCAS and the adopted version used in this study showed sufficient reliability and internal consistency (Adjekum, 2014; Adjekum et al., 2015).

\section{Procedure}

After Institutional Review Board (IRB) approval was obtained from all participating programs, purposeful sampling was used to target the ATC, AVMgt, and UAS students enrolled at the five selected programs. An email including the survey link was distributed. The survey was open for four weeks during the spring semester of 2015. The estimated population was approximately five hundred $(N=500)$.

Respondents were asked to rate perceptions on items of the various measurement constructs in the survey instrument using a five-point Likert scale ( $1=$ strongly disagree $-5=$ strongly agree). Respondents were given the option to provide demographic information, such as gender, age, and education level to enhance data analysis. Finally, respondents were asked the number of times they had self-reported safety issues in their programs (reporting frequency).

The quantitative data was uploaded and coded appropriately into IBM SPSS $® 23$ statistical software package for analysis. The multi-item scales were the independent variables and indicators of safety culture perceptions. The dependent variable was Safety Behavior (frequency of voluntary self- reporting of safety issues). Regarding the mediating variables for the last two questions, Reporting System, and Safety Values were used as observed endogenous variables. 


\section{Sample Size, Power Analysis, and Response Rate}

A power analysis and sample size determination was based on Boomsma and Hoogland (2001) recommendations that 200 cases constitute a reliable sample size for a correct model in CFA and SEM analysis; one in which any problem related to power analysis is less likely to occur. Based on the estimated number of invitations sent to respondents for the study $(N=500)$ and the one hundred and sixteen $(n=116)$ responses completed beyond the consent page and used for analysis, the response rate was about $24 \%$ using a Survey Monkey® response rate calculator.

\section{Results}

\section{Demographics}

Out of the one hundred and sixteen $(n=116)$ responses completed and used for the analysis, ninety-two male (79.3\%) and twenty-one female (18.1\%) respondents submitted useable data for analysis. Three respondents did not disclose their gender (2.6\%). The respondents comprised of six educational level groups of freshmen, sophomore, juniors, seniors, graduate students, and others. Juniors were the modal group followed by seniors. The smallest educational group was graduate students. Forty-three respondents were ATC majors (37.1\%), thirtynine respondents were UAS majors (33.6\%), thirty-three were AVMgt majors $(28.4 \%)$, while one respondent did not identify with any major.

A breakdown of the respondents by colleges showed that, out of the five aviation colleges, $10.3 \%$ of the respondents were from the first college; approximately $22 \%$ were from the second college, $28 \%$ from the third college, $11 \%$ from the fourth college and approximately $28 \%$ from the fifth college. Approximately $1 \%$ of the respondents did not identify with any college. It was interesting to note that colleges 3 and 5 have both attained the status of active conformance which is last stage of the FAA voluntary SMS program for aviation training organizations. The active conformance level is attained when the Certificate Maintenance Team (CMT) and the SMS Program Office (SMSPO) of the FAA acknowledge full implementation of the certificate holder's SMS. By this stage, the certificate holder is expected to use and continually improve its safety management processes (FAA, 2015). Table 1 and Table 2 provide a summary of all the demographic data used in the analysis. 
Table 1

Demographic variables of Gender and Educational Level Group

\begin{tabular}{lll}
\hline \multicolumn{1}{c}{ Variable } & Value & Percentages (\%) \\
\hline Gender & 92 & 79.3 \\
Male & 21 & 18.1 \\
Female & 3 & 2.6 \\
No response & 116 & 100.0 \\
Total & & \\
& & \\
Educational Level & 21 & 18.4 \\
Freshmen & 16 & 14.0 \\
Sophomore & 40 & 35.1 \\
Junior & 24 & 21.1 \\
Senior & 11 & 9.6 \\
Graduate Students & 4 & 3.5 \\
No response & 116 & 100.0 \\
Total & &
\end{tabular}

Note. Percentages are approximate values.

\section{Internal Consistency and Construct Validity}

An Exploratory Factor Analysis (EFA) (Principal Axis Factoring) was conducted on each scale using a varimax rotation. An EFA is a statistical method used to find a small set of unobserved variables (also called latent variables, or factors) which may account for the covariance among a larger set of observed variables (Steven, 2002). A factor is an unobservable variable that is assumed to influence observed variables. Items with strong loading on factors were extracted from each set of items in the subscales.

Strongly loaded items on each factor were identified using the factor loadings and the scree plot of the SPSS ${ }^{\circledR}$ output. Cut-off factor loading values of greater than 0.5 and Eigen values greater than 1 were used. The factors and percentage of variance explained by the Eigen values were determined and are shown in Table 3. The factor loadings are shown in Appendix A. Internal consistency and reliability of the scales were determined with the Cronbach's Alpha 
test in the SPSS 23® software package. Generally, for social sciences, an alpha $(\alpha)$ of .70 and above indicates high internal consistency (Stevens, 2002; Fields, 2009).

Table 2

Demographic Variables of College and Age Group

\begin{tabular}{|c|c|c|}
\hline Variables & Values & Percentages $(\%)$ \\
\hline \multicolumn{3}{|l|}{ College/Institution } \\
\hline 1 & 12 & 10.3 \\
\hline 2 & 26 & 22.4 \\
\hline 3 & 32 & 27.6 \\
\hline 4 & 13 & 11.2 \\
\hline 5 & 32 & 27.6 \\
\hline No response & 1 & 0.9 \\
\hline Total & 116 & 100.0 \\
\hline \multicolumn{3}{|l|}{ Age Group } \\
\hline Below 20 & 24 & 20.7 \\
\hline $20-30$ & 82 & 70.7 \\
\hline $31-40$ & 6 & 5.2 \\
\hline $41-50$ & 2 & 1.7 \\
\hline No response & 2 & 1.7 \\
\hline Total & 116 & 100.0 \\
\hline \multicolumn{3}{|l|}{ Academic Major } \\
\hline Air Traffic Control (ATC) & 43 & 37.1 \\
\hline Unmanned Aircraft System (UAS) & 39 & 33.6 \\
\hline Aviation Management (AVMgt) & 33 & 28.4 \\
\hline No response & 1 & 0.9 \\
\hline Total & 116 & 100.0 \\
\hline
\end{tabular}

Note. Percentages are approximate values.

All the items in the various scales showed adequate reliability above the .70 threshold. Descriptive statistics on the summed items in each scale were conducted. The results were determined to be consistent with the assumptions of normally distributed data. The assumption of normality was confirmed based on histograms with normality plot. The kurtosis and skewness values of the descriptive statistics tables were in the acceptable range of -1 to +1 . Details of the sample size, mean, SD and other pertinent data are shown in Table 3.

In order to determine how well the measurement models for the various scales matched the data; a first-order confirmatory factor analysis (CFA) was 
conducted using all the items extracted under factors for RS, RF, SV, RF, and ADSR. Additionally, the CFA was used to analyse the validity and reliability of the safety culture scales using a structural equation model (SEM). SPSS AMOS 23® software was used to evaluate the measurement models and goodness of fit to the observed data underlying the safety culture constructs.

A CFA allows researchers to test hypotheses about a particular factor structure (e.g., factor loading between the first factor and first observed variable is zero). Unlike an EFA, a CFA produces several goodness-of-fit measures to evaluate the model but do not calculate factor scores (Brown, 2006). A large class of omnibus tests exists for assessing how well the model matches the observed data, and the chi-square $\left(\chi^{2}\right)$ is a classic goodness of fit measure to determine overall model fit. However, the chi-square is sensitive to sample size, and it becomes difficult to retain the null hypothesis as the number of cases increases (Kline, 2005). The $\chi^{2}$ test may also be invalid when distributional assumptions are violated, leading to the rejection of good models or the retention of bad ones (Brown, 2006; Steven, 2002).

Another commonly reported statistic is the Root Mean Square Error of Approximation (RMSEA), a measure of fit introduced by Steiger and Lind (1980), who suggested that a value of the RMSEA of 0.05 or less indicates a close fit of the model in relation to the degrees of freedom. Another test statistics is the Comparative Fit Index (CFI) that evaluates "the fit of a user-specified solution in relation to a more restricted, nested baseline model, in which the covariance among all input indicators are fixed to zero or no relationship among variables is posited" (Brown, 2006, p. 86). CFI ranges from 0, for a poor fit, to 1 for a good fit. Finally, the Tucker-Lewis Index (TLI), is another index for comparative fit that "includes a penalty function for adding freely estimated parameters" (Brown, 2006, p. 85). According to Brown (2006), TLI may be interpreted in a similar fashion as CFI, but can have a value outside of the range of 0 to 1 .

$\mathrm{Hu}$ and Bentler (1999) provided rules of thumb for deciding which statistics to report and choosing cut-off values for declaring significance. When RMSEA values are .05 or below, and CFI and TLI are .95 or greater, the model may have a reasonably good fit. Therefore, it is recommended to not only report $\chi^{2}$ but RMSEA and CFI/TLI. In the case of the chi-square goodness of fit, if the appropriate distributional assumptions are met and the specified model is correct, then the values of the $p$-values is the approximate probability of a chi-square statistic. 


\section{Research Questions}

Question One. What is the validity of the measurement models for the safety culture scales: Safety Value (SV), Safety Fundamental (SF), Response and Feedback $(R F)$, Reporting System (RS), and Aviation Department Safety Record $(A D S R)$ ?

A first-order confirmatory factor analysis (CFA) and structural equation model (SEM) was used to measure and determine the validity of the measurement models derived from all the items extracted under factors for RS, RF, SV, RF, and ADSR using the initial EFA. The results suggest that all of the $p$-values of the CFA were above the .05 threshold and denotes a departure of the data from the model (not significant at the .05 level). However, since the sample size was low $(<200)$ for a CFA, low power could potentially have led to non-significant chi-square values (Kline, 2005).

The goodness of fit indices for the CFA were all deemed reasonably good for all the models and are highlighted in Table 3. However, the almost "perfect fit" for the RS and RF scales may indicate a potential for configural invariance, even though the $p$-value of the chi-square value is non-significant. Appendix A also shows the Structural Equation Models (SEM) of the first-order CFA of the scales, RS, SF, SV, and RF.

Question Two. What is the magnitude of the overall perceptions of respondents on the safety culture scales: Safety Values (SV), Safety Fundamentals $(S F)$, Response and Feedback (RF), Reporting System (RS), and Aviation Department Safety Record (ADSR)?

The researchers sought to find out the general trend and directions of the magnitude of the perceptions of respondents on the safety culture within their programs and how it invariably affects their perceptions about the safety occurrence potential within the program. After the preliminary assessment of the data for normality, construct validity and reliability, the remaining items retained under the various scales were summed. Then the mean value of these scores were determined and compared to the baseline Likert scale value of three (Neutral) on the five-point scale.

Values of negatively worded items were reverse-coded and standardized to comparable readings. In general, it was assumed any mean score that was greater than the neutral value of three was acceptable. As the mean value approached the 
maximum value of 5, it suggested an extremely favorable perception of that scale item by respondents. The results indicated the highest perception mean score was on Safety Value $(M=4.05, S D=.778)$ and the lowest perception mean score was on Response and Feedback $(M=3.54, S D=.727)$.

Table 3

Values of Exploratory Factor Analysis, Confirmatory Factor Analysis, Reliability and Descriptive Statistics of Safety Culture Measurement Constructs.

\begin{tabular}{llllll}
\hline \multicolumn{5}{c}{} & \multicolumn{2}{c}{ Variables } \\
\cline { 5 - 6 } & $\mathrm{RS}$ & $\mathrm{RF}$ & $\mathrm{SV}$ & $\mathrm{SF}$ & ADSR \\
\hline$n$ & 116 & 116 & 116 & 116 & 116 \\
Mean & 3.59 & 3.54 & 4.05 & 3.84 & 3.38 \\
Std. Deviation & .765 & .727 & .779 & .780 & 1.10 \\
Variance & 41.35 & 51.57 & 58.46 & 68.65 & - \\
explained $(\%)$ & .80 & .82 & .76 & .85 & .90 \\
Cronbach's $\alpha$ & 5 & 4 & 4 & 4 & 3 \\
Items in Scale & 5 & $(2)=1.256$ & $(2)=21.74$ & $(2)=14.30$ & - \\
Chi-square: $X^{2}(\mathrm{n})$ & $(5)=2.751$ & .992 & .852 & .931 & - \\
CFI & .983 & 1.00 & .863 & .940 & - \\
TLI & 1.00 & .000 & .029 & .023 & - \\
RMSEA & .000 & .534 & .415 & .367 & - \\
$p$-value & .737 & & & &
\end{tabular}

Interestingly,these results contrasted earlier findings among commercial flight students and instructors from the same population, where the highest perception mean score was on Safety Fundamentals (4.15) and the lowest perception mean score was on Safety Value (3.45) (Adjekum et al., 2015). However, both scale items were above the neutral point of 3 . In terms of the safety occurrence potential perceptions, that was assessed through ADSR, the mean perception value was below the neutral value of three, and not relatively good $(M=2.62, S D=1.108)$. The mean scores of all the scale items are shown in Figure 1. 


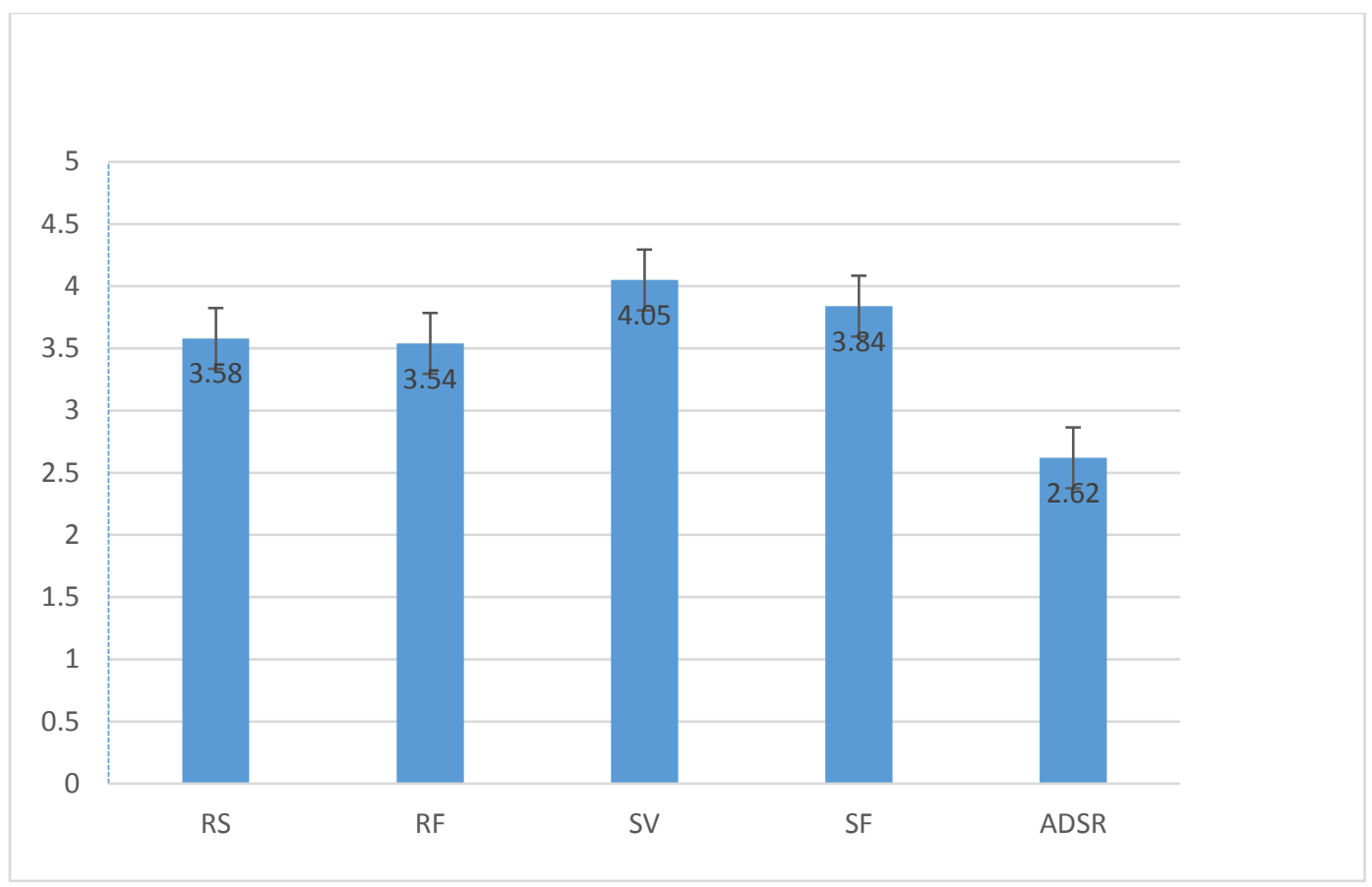

Figure 1. Magnitude of perceptions of respondents of safety culture scales: Reporting System (RS), Response and Feed-back (RF), Safety Value (SV),Safety Fundamentals (SF) and Aviation Department Safety Records (ADSR).

Question Three. What are the strengths of relationship between respondent perceptions on the safety culture scales: Safety Values (SV), Safety Fundamentals $(S F)$, Response and Feedback (RF), Reporting System (RS), Aviation Department Safety Record (ADSR), demographic variables, and frequency of self- reporting (REPFREQ) safety issues by respondents?

A Pearson's bivariate test of correlations was used to establish the strengths of relationship between the safety culture perception of respondents and their safety reporting behavior (REPFREQ). This analysis was conducted, to find out variables that were linearly related, and could potentially become viable predictors in the subsequent confirmatory factor analysis. The result suggested that the perceptions of respondents on the reporting system (RS) was positive and significantly correlated with their perceptions on the response and feedback (RF) in their programs, $r(116)=.75, p<.01$. Another significant positive correlation existed between RF and SF, $r(116)=.75, p<.01$.

A strong and positively significant relationship existed between RS and SF, $r(116)=.73, p<.01$. A relatively weak significant negative correlation existed between Age and SV, $r(116)=-.20, p<.05$. There existed a strong statistically 
significant correlation between SV and SF, $r(116)=.54, p<.01$. There was also a weak statistically significant positive relationship between RS and Safety Reporting Frequency (REPFREQ), $r(116)=.23, p<.05$. There existed a relatively weak statistically significant relationship between REPFREQ and SF, $r(116)=.20$, $p<.05$. Finally, there existed a moderate statistically significant negative relationship between REPFREQ and AGE, $r(116)=-.30, p<.01$. Details of the results of the correlations are summed up in Table 4.

Table 4

Pearson's Bivariate Correlations of all the scales in the study

Ed Safety

Reporting

\begin{tabular}{lllllllll} 
Variables & $\mathrm{RS}$ & $\mathrm{RF}$ & $\mathrm{SV}$ & $\mathrm{SF}$ & ADSR Age & Gender level Frequency \\
\hline
\end{tabular}

RS $\quad 1$

$\mathrm{RF} \quad .75^{* *} \quad 1$

SV $\quad .34^{* *} \quad .33^{* *} 1$

SF $\quad .73^{* *} \quad .75^{* *} \quad .54^{* *} \quad 1$

$\begin{array}{lccccccccc}\text { ADSR } & -.05 & -.03 & .15 & -.08 & 1 & & & \\ \text { Age } & -.08 & -.06 & -.20^{*} & -.19^{*} & .04 & 1 & & & \\ \text { Gender } & -.152 & -.16 & -.14 & -.20^{*} & -.05 & .10 & 1 & & \\ \text { Education } & -.11 & -.14 & -.17 & -.15 & .15 & .71^{* *} & .06 & 1 & \\ \begin{array}{l}\text { level } \\ \text { Safety }\end{array} & & & & & & & & & \\ \begin{array}{l}\text { Reporting } \\ \text { Frequency }\end{array} & .23^{*} & .20^{*} & .01 & .13 & -.12 & -.30^{* *} & -.11 & -.16 & 1\end{array}$

* Correlation is significant at the 0.05 level (2-tailed)

** Correlation is significant at the 0.01 level (2-tailed).

Question Four. What is the relationship between age and frequency of selfreporting safety issues (REPFREQ) when mediated by Reporting System (RS)?

A mediation analysis was performed using the Baron and Kenny (1986) causal-step approach and the Preacher and Hayes (2008) bootstrapped confidence 
interval for $a b$ indirect effect procedure using the SPSS AMOS® 23 software package. The path analysis helped to establish predictive causal path and relationships between some exogenous causal variables such as AGE and an endogenous outcome variable such as REPFREQ, using an endogenous mediating variable RS. The path models also helped to determine the causal path coefficients for the variables under investigations. The maximum likelihood estimates, standardized regression weights, critical ratios, total, direct and indirect effects were determined. Finally, the $p$-value was also determined to find out whether the hypothesis under examination was supported.

The initial causal exogenous variable was age groups of respondents (AGE) and the observed endogenous variable was the frequency of self-reporting (REPFREQ) safety events while the mediating endogenous variable was a perception on the safety reporting system (RS) in the program. Preliminary data screening suggested that there were no serious violations of the assumptions of linearity or normality. All the coefficients reported are standardized and the twotailed statistical significance criterion was .05.

Since the hypothesized model had zero degrees of freedom, the chi-square statistic was zero, implying that the model fit the data perfectly, and consequently, no probability level could be assigned to the chi-square statistic. Since each variable had a direct path to every other variable in this analysis; the chi-square for the model fit was zero and the implication was that the path coefficient constructs the variances and covariance among the observed variables. The appropriate distributional assumptions were met and the specified model was assumed correct. The $p$-value obtained was the approximate probability of getting a chi-square statistic as large as the chi-square statistic obtained from the current set of data.

The direct effect of AGE variable on REPFREQ was statistically significant $(\beta=-.288, t[112]=-2.791, p<.01)$ and implies that when AGE goes up by 1 standard deviation, REPFREQ goes down by 0.29 standard deviations. The result indicates that the hypothesis $H_{1}$ was supported. Even though AGE was not significantly predictive of the mediating variable $\mathrm{RS}(\beta=-.108, t[112]=-.862, p$ $=.276)$, when AGE was controlled, there was a significant direct effect of RS on REPFREQ $(\beta=.194, t[112]=2.153, p<.05)$. The variance in REPFREQ was accounted for by $15.4 \%$ of the predictor AGE.

The standardized indirect (mediated) effect of AGE on REPFREQ by RS, was -.019 and not significantly different from zero at the 0.05 level $(p=.307)$. A bootstrap approximation was obtained from the indirect procedure in SPSS AMOS script (Preacher \& Hayes, 2008), by requesting 5,000 samples. The two-sided bias- 
corrected confidence intervals (CI) was created for $(a b)$ and were (-.071 to .026) at the $95 \% \mathrm{CI}$.

Even though not statistically significant, the implication is that, due to the indirect (mediated) effect of AGE on REPFREQ by RS, when AGE goes up by 1 standard deviation, REPFREQ goes down by 0.019 standard deviations. This is in addition to any direct (unmediated) effect that AGE may have on REPFREQ. This result suggests that RS did not have significant mediating effect on the causal path between AGE and REPFREQ and the hypothesis $H_{2}$ was not supported.

Question Five. What is the relationship between Safety Fundamentals (SF) and frequency of self-reporting of safety issues when mediated by Safety Values $(S V)$ ?

Another mediation analysis was performed using the Baron and Kenny (1986) causal-step approach and the Preacher and Hayes (2008) bootstrapped confidence interval for $(a b)$ indirect effect procedure using the SPSS AMOS $® 23$ software package. The path analysis helped to establish predictive causal path and relationships between the exogenous causal variable SF and an endogenous outcome variable such as REPFREQ, using an endogenous mediating variable SV. The results suggest a significant direct effect of SF on $\mathrm{SV}(\beta=.567, t[113]=6.870$, $p<.001$ ). The standardized total (direct and indirect) effect of SF on SV was .567. That is, due to both direct (unmediated) and indirect (mediated) effects of SF on SV, when SF goes up by 1 standard deviation, SV goes up by 0.567 standard deviations.

There was also a significant direct effect of SF on REPFREQ ( $\beta=.214, t$ $[113]=1.438, p<.05)$. However, there was no significant mediating effect of SV on REPFREQ $(\beta=-.139, t[113]=2.153, p=.215)$. A bootstrap approximation was obtained from the indirect procedure in SPSS AMOS script (Preacher \& Hayes, 2008), by requesting 5000 samples. The two-sided bias- corrected confidence intervals (CI) was created for $(a b)$ and were (-.260 to .042) at the $95 \% \mathrm{CI}$.

The standardized indirect (mediated) effect $(a b)$ of the variable SF on REPFREQ was -.084. The result implies that due to the indirect (mediated) effect of SF on REPFREQ by SV, when SF goes up by 1 standard deviation, REPFREQ goes down by 0.084 standard deviations. This is in addition to any direct (unmediated) effect that SF may have on REPFREQ. The indirect (mediated) effect of SF on REPFREQ, by SV was not significantly different from zero at the 0.05 level $(p=.205)$. 
The total effect of SF on REPFREQ, when mediated by SV was not significant, but the direct effect of SF on REPFREQ was significant and the hypothesis $\mathrm{H}_{3}$ was supported. The results also suggest that the relationship between the variables Safety Fundamentals and reporting frequency when mediated by Safety Values $\left(\mathrm{H}_{4}\right)$ was not supported. The details of all the analysis are shown in Table A1 in Appendix A. The path analysis/structural equation models of the study are also shown in Appendix A.

\section{Discussion and Conclusion}

The confirmatory factor analysis (CFA) approach was used to validate the data obtained after the exploratory factor analysis. This was to test the underlying theory relating observed survey instrument items that measure latent scales such as Safety Fundamentals and Reporting Systems in collegiate aviation programs. Even though the sample size was low $(<200)$ for CFA, and the chi-square values were non-significant, other alternative goodness of fit measures were used to test the construct validity of the scales (Kline, 2005). The goodness of fit indices for the CFA were all reasonably good for all the models and the scales were recommended as tools in assessing the safety culture within collegiate aviation.

The lowest perceptions mean score was with the scale Response and Feedback. A perception of potential safety occurrences was assessed through ADSR and also had low scores. Comparing these findings to the safety culture perceptions of traditional flight students within the same population (Adjekum et al., 2015), these results suggest that various academic majors have different areas of emphasis when examining the safety culture in collegiate aviation programs. The results suggest that, although non-flight majors may be distal from the direct flight operational activities, their perceptual perspectives may be informed by the general trends, attitudes and perceived safety values in the entire collegiate program.

Even though these non-flight majors may not be directly involved in the flight operational activities such as active flying, interaction with both flight students and operational personnel during ground school may also influence overall safety culture perceptions. Under such circumstances, even though these perspectives may not be real and may be based on third-party information about safety lapses and occurrences in the program, such trends could skew their perceptions on the likelihood of safety occurrences and violations of regulations by operational personnel.

Another factor, that could explain these results, may be the situation where non-flight majors are not included in flight safety risk management training as 
compared to traditional flight students during SMS implementation. Another possible reason could be the scope and coverage of safety promotional resources for these non-flight majors, which may sometimes be limited, based on the size and complexity of the SMS within the respective collegiate programs. These trends may affect how respondents build capacity to identify hazards and file a safety report. This should be a reason why collegiate aviation program managers need to include and involve all personnel whether on active flight status or in non-flight roles in safety promotion and education programs.

The results suggest that when Response and Feedback from safety personnel are not proactive, non-flight majors interest to self-report safety issues may decline. These non-flight majors may not spend time to fill out hazard reports, since it might be deemed as a waste of time, and in the long run, their effort unappreciated. That scenario may also heighten perceptions that risk mitigation and control strategies are not effective. These respondents may then perceive that their program's susceptibility to risk of safety occurrences and possible violations are high, with an attendant increased accident potential.

The lack of feedback from safety professionals or operational managers has been highlighted as inhibiting the willingness of personnel to report incidents (Reason, 2008; Stolzer, Halford \& Goglia, 2011). This is actually coupled with the ever present challenge of factors that inhibit reporting, including fear of blame, time pressure, resource constraints, the perception that reporting is unnecessary (Benn et al., 2009). Another challenge has been the lack of clear definitions as to what constitutes a reportable incident.

Improving the expeditiousness of responses and feedback in the collegiate aviation operations should be an intrinsic part of the safety reporting system and may increase reporting rates, since the impact of safety feedback on the development of a positive safety culture within collegiate aviation operations should not be under-estimated (Adjekum, 2014). The significantly positive predictive relationship between reporting system and reporting frequency, underscores the importance of a proactive safety reporting system.

Getting the content of feedback right in terms of the message it conveys, regarding how incident data may be used, the level of anonymity provided to reporters and the potential consequences of disclosing errors and near misses through reporting are all critical issues that may impact upon reporting culture (Benn et al., 2009). Feedback that promotes a systemic, rather than individual view on the causes of failure may underpin the development of a safety-conscious and 
just culture within an organization and that could promote the future reporting of failures in collegiate aviation programs (Dekker, 2007).

The strong correlation between the perceptions of respondents on the reporting system and response and feedback buttress the points raised on the need for greater diligence and awareness of the feedback mechanism for safety reporting system, if collegiate aviation programs want to enhance their safety reporting culture. The significantly predictive nature of the AGE variable on reporting frequency (negative) corroborates with earlier findings of Adjekum et al., (2015) on safety culture assessments among flight students and flight instructors in collegiate aviation programs. The results suggest that relatively younger respondents have a higher rate of self-reporting safety issues as compared to older respondents.

In an era, where collegiate aviation programs are gradually becoming demographically diverse, program managers may miss the mark, if the focus of safety programs is only aimed at targeting new, young and inexperienced operational personnel while paying minimal attention to the safety behavior of older experienced students (Adjekum et al., 2015). The results corroborate earlier findings that relatively older and matured collegiate aviation students, may sometimes by virtue of aviation experiences become complacent, overlook hazards, and even regard minor safety issues as normal (Adjekum et al., 2015).

Sometimes, some of the older students may not have the alacrity to report safety issues due to relatively numerous personal responsibilities and demanding schedules, coupled with the complicated procedures for reporting hazards and identified risk in some collegiate aviation programs. Older respondents may feel slighted and eventually not bother to self-report if previously submitted reports did not elicit productive and reasonable feedback from program safety staff. That may rather increase the level of apathy and slacken the effective reporting of future safety issues. The results suggest that most of the respondents were within the 20 30 -year age bracket and it may be expedient to focus more safety risk management resources among respondents in this age range. In summary, safety reporting programs and promotions should be developed and targeted towards all the different age groupings within the collegiate aviation environment.

The strong significant direct effect of Safety Fundamentals on Safety Values underscores the need by collegiate aviation management to confirm that safety performance criteria and outcomes are purposefully aligned to stated safety objectives. The aim of SMS and other progressive safety initiatives is to ensure that safety compliance and performance indicators far exceed regulatory requirements 
and that is reflected in continuous improvement of safety processes such as SOPs, training procedures and checklist updates and usage by operational personnel.

For non-flight majors, an excellent and performance-oriented ground school and aviation academic curriculum, that highlights the importance of the safety vision and policy of the collegiate program, may help to enhance their perceptions on both the safety value and safety fundamentals in the program. When collegiate aviation programs have a robust Safety Fundamentals, which encourages strict compliance with SOPs and regulatory standards by operational personnel, the benefits may include a reduction in the accident potential and improvement of the safety culture perceptions of both students and personnel.

It was rather interesting that perceptions on Safety Values were not a significant predictor of reporting frequency. This finding suggests that sometimes there may be other confounds and a socio-cultural bias that may influence individual concepts of what constitutes a value and especially safety values. Also reporting frequency may be a function of hazard observability and identification, coupled with the motivation to improve an operational safety system (Yates, 2015). If non-flight majors are not well versed or involved in activities that promote the safety values of the program, the chances of observing and identifying hazards may be limited (ICAO, 2013; Stolzer, Halford \& Goglia, 2011; Yates, 2015). These students may not even be aware of the hazards and risks within an aviation department.

The analyses found out that the scale, Safety Fundamentals was a significant positive predictor of reporting frequency. The scale underscores earlier suggestions that SOPs, checklists, written procedures, and compliance with the regulated aspect of safety, cut across the spectrum of collegiate aviation. If the rationale for any collegiate program is to ensure continuous safety improvement, there should be acceptance and responsibility for operational safety from all personnel. Personnel at all levels may contribute to the building up of a proactive safety culture through constant and periodic safety reporting. Finally, well informed personnel may create a proactive safety working environment, which invariably promotes a positive safety culture (Yates, 2015).

Some of the inherent limitations of this study included the relatively small sample size, which may have affected the results, especially for the CFA and SEM analysis, where most of the test is sensitive to sample size. Even though the bootstrapping technique was used, a larger sample size may have given better power for the hypotheses testing. The survey was anonymous and as such it was difficult to ascertain whether respondents took it more than once. Even though within the study 
period there were no reported major safety mishaps in any of the colleges that took part in this study normally the perceptions of respondents on safety culture could sometimes be influenced by sudden and drastic safety events within the collegiate aviation community.

In terms of generalization of research findings and applicability, the researchers cautiously limit the findings of this study to collegiate aviation programs with similar scale and complexity in terms of voluntary SMS implementation in the US. This study however, is the first of its kind relating to non-flight majors in collegiate aviation and establishes a benchmark for further study. The study also underscores the need for collegiate aviation programs to invest in proactive safety programs such as SMS that target all students. It is hoped that this study will assist to sustain the human resource and capital base of the aviation industry world-wide through proactive, performance-based and safetyoriented aviation training. A future study of either longitudinal or concurrent triangulation is highly recommended and should have a wider scope in terms of sample size, colleges, and international reach. 


\section{References}

Adjekum, D. K. (2014) Safety culture perceptions in a collegiate aviation program: A systematic assessment, Journal of Aviation Technology and Engineering, 3(2). http://dx.doi.org/10.7771/2159-6670.1086

Adjekum, D. K., Keller, J., Walala, M., Young, J. P., Christensen, C., DeMik, R. J., \& Northam, G. (2015). Cross-sectional assessment of safety culture perceptions and safety behavior in collegiate aviation programs in the United States. International Journal of Aviation, Aeronautics, and Aerospace, 2(4). http://dx.doi.org/10.15394/ijaaa.2015.1074

Baron, R. M., \& Kenny, D. A. (1986). The moderator-mediator variable distinction in social psychological research - Conceptual, strategic, and statistical considerations. Journal of Personality and Social Psychology, 51(6), 1173-1182.

Benn, J., Koutantji, M., Wallace, L., Spurgeon, P., Rejman, M., Healey, A., \& Vincent, C. (2009). Feedback from incident reporting: Information and action to improve patient safety. Quality Safety Health Care, 18. 11-21 doi: 10.1136/qshc.2007.024166.

Bollen, K., \& Long, J. (1993). Testing structural equation models. Newbury Park, California: Sage Publications.

Boomsma, A., \& Hoogland, J. J. (2001). Structural equation modeling: Present and future: A Festschrift in honor of Karl Jöreskog. Chicago: Scientific Software International.

Brown, T. A. (2006). Confirmatory factor analysis for applied research. New York: Guilford Press

Chen, C.-F.-C. (2014). Measuring the effects of safety management system practices, morality leadership and self-efficacy on pilots' safety behaviors: Safety motivation as a mediator. Safety Science, 62(14), 376-385. doi: http://dx.doi.org/10.1016/j.ssci.2013.09.013

Cooper, M. D. (2000). Towards a model of safety culture. Safety Science, 36(2), 111-136. doi: http://dx.doi.org/10.1016/S0925-7535(00)00035-7 
Creswell, J. W. (Ed.). (2009). Research design: Qualitative, quantitative, and mixed method approaches (3rd ed.). Thousand Oaks, CA: Sage Publications, Inc.

DeJoy, D., Schaffer, B., Wilson, M., Vandenberg, R., \& Butts, M. (2004). Creating safer workplaces: Assessing the determinants and role of safety climate. Journal of Safety Research, 35(1), 81-90. doi: 10.1016/j.jsr.2003.09.018

Dekker, S.W.A. (2007). Just Culture: Balancing safety and accountability. Burlington, VT : Ashgate Publishing

Dillman, B., Voges, J., \& Robertson, M. (2010). Safety occurrences: Student perceptions regarding failure to report. Journal of Aviation Management and Education, 1. Retrieved from http://www.aabri.com/manuscripts/09261.pdf

Drinkwater, L., \& Molesworth, B.R.C. (2010). Pilot see, pilot do: Examining the predictors of pilots' risk management behavior. Safety Science 48, 1445 1451. doi: http://dx.doi.org/10.1016/j.ssci.2010.07.001

Federal Aviation Administration. (2012). Safety management system for pilot schools and training centers. Washington, D.C.: Air Traffic Organization NextGen \& Operations Planning, Office of Research and Technology Development.

Federal Aviation Administration. (2015). Safety management system voluntary program guide (AFS-900-002-G201) [PDF]. Washington D.C.: Federal Aviation Administration. Accessed from: University of North Dakota Aviation Safety Department.

Field, A. (2009). Discovering statistics using SPSS (3rd ed.). London: SAGE Publications, Ltd.

Freiwald, D., Lenz-Anderson, C., \& Baker, E. (2013). Assessing safety culture within a flight training organization. Journal of Aviation/Aerospace Education and Research, 22(2). Retrieved from http://commons.erau.edu/db-applied-aviation/2 
Griffin, M. A., \& Neal, A. (2000). Perceptions of safety at work: A framework for linkingsafety climate to safety performance, knowledge and motivation. Journal of Occupational Health Psychology, 5(3), 347-358. doi: 10.1037//1076-8998.5.3.347

Hu, L., \& Bentler, P.M. (1999), Cutoff criteria for fit indexes in covariance structure analysis: Conventional criteria versus new alternatives. Structural Equation Modeling, 6, 1-55.

Hudson, P. (2001). Aviation safety culture: Presentation at Safeskies 2001, Canberra, Australia, 31 October-2 November. Retrieved from : http://www.faa.gov/about/initiatives/maintenance_hf/losa/publications/me dia/lewis_christopher_(2008)_sms_developmenteffectivesafetyculture.pdf

Hunter, D. R. (2006). Risk perception among general aviation pilots. The International Journal of Aviation Psychology, 16(2), 135-144. doi: http://dx.doi.org/10.1207/s15327108ijap1602_1

International Civil Aviation Organization. (2009). Safety Management Manual (SMM) (Doc.9859 [2nd ed.]) [PDF]. Montréal: Author.

International Civil Aviation Organization (2013). Safety Management Manual, Doc 9859 AN/474 ( $3^{\text {rd }}$ ed.). Montréal: Author.

Kline, R. B. (2005). Principles and Practice of Structural Equation Modeling (2nd ed.). New York: Guilford.

Mearns, K., Whitaker, S., \& Flin, R. (2003). Safety climate, safety management practices, and safety performance in offshore environments. Safety Science, 41, 641-680. doi: http://dx.doi.org/10.1016/S09257535(02)00011-5

Molesworth, B.R.C., \& Chang, B. (2009). Predicting pilots risk-taking behavior through an implicit association test. Human Factors 51(6), 845-857. doi: http://dx.doi.org/10.1177/0018720809357756 
Preacher, K. J., \& Hayes, A. F. (2004). SPSS and SAS procedures for estimating indirect effects in simple mediation models. Behavior Research Methods, Instruments, \& Computers, 36, 717-731. doi:10.3758/BF03206553

Preacher, K, J., \& Hayes, A, F. (2008). Asymptotic and resampling strategies for assessing and comparing indirect effects in multiple mediator models.

Behavior Research Methods 40(3), 879-891. doi: 10.3758/BRM.40.3.879

Steiger, J. H., \& Lind, J. (1980), "Statistically-based tests for the number of common factors," Paper presented at the Annual Spring Meeting of the Psychometric Society, Iowa City.

Stevens, J. (2002). Applied Multivariate statistics for the social sciences $\left(4^{\text {th }} \mathrm{ed}\right.$.) Hillsdale, NJ: Erlbaum.

Stolzer, A.J., Halford, C.D., \& Goglia, J.J. (2011). Implementing safety management systems in Aviation. Surrey, UK: Ashgate Publishing.

Reason, J. T. (2008). The human contribution: unsafe acts, accidents and heroic recoveries. Burlington, VT: Ashgate Publishing.

Yates, W. D. (2015). Safety professional's reference and study guide (2 ${ }^{\text {nd }}$ ed.). Boca Raton, FL: CRC Press.

Zohar, D., \& Luria, G. (2004). Climate as a social-cognitive construction of supervisory safety practices: Scripts as proxy of behavior patterns. Journal of Applied Psychology, 89(2), 322-333. doi: 10.1037/0021-9010.89.2.322 


\section{Appendix}

Table A1

Standardized Regression Estimates, Effects, T-values, $P$-values and Hypotheses

\begin{tabular}{|c|c|c|c|c|c|c|c|c|c|}
\hline Dependent & & Predictor & $\beta$ & $\begin{array}{c}\text { Indirect } \\
\text { Effect }\end{array}$ & $\begin{array}{l}\text { Direct } \\
\text { Effect }\end{array}$ & $\begin{array}{l}\text { Total } \\
\text { Effect }\end{array}$ & $t$-value & $P$-value & Hypothesis \\
\hline $\mathrm{RS}$ & $<-$ & AGE & -.108 & - & -.108 & -.108 & -.862 & .276 & $\begin{array}{c}\text { Not } \\
\text { Supported }\end{array}$ \\
\hline REPFREQ & $<-$ & AGE & -.288 & -.019 & -.269 & -.288 & -2.791 & $* * *$ & Supported \\
\hline REPFREQ & $<-$ & $\mathrm{RS}$ & .194 & - & .194 & .194 & 2.153 & $.034 *$ & Supported \\
\hline SV & $<-$ & SF & .567 & - & .567 & .567 & 6.870 & $* * *$ & Supported \\
\hline REPFREQ & $<-$ & SF & .214 & -.046 & .214 & .168 & 1.438 & $.048 *$ & Supported \\
\hline REPFREQ & $<-$ & SV & -.139 & - & -.082 & -.082 & -1.268 & .473 & $\begin{array}{c}\text { Not } \\
\text { Supported }\end{array}$ \\
\hline
\end{tabular}

Note: $* \mathrm{p}<.05, * * * \mathrm{p}<.001$ 


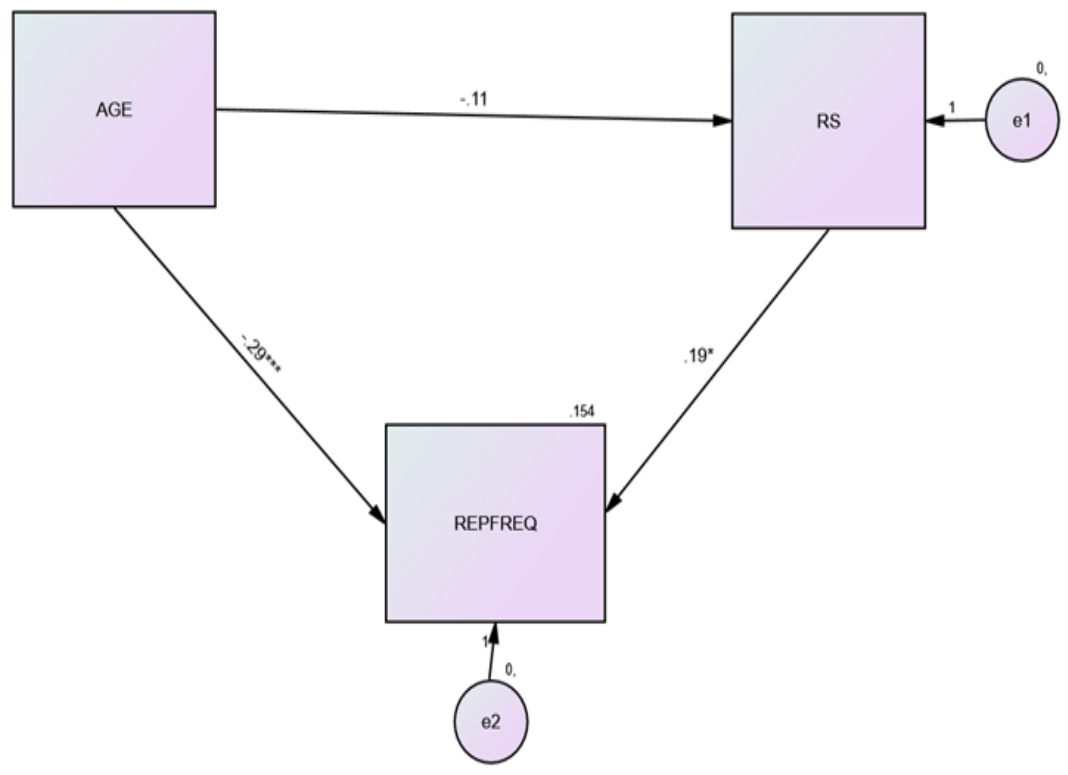

Figure A1. Final Path Model for Mediation Analysis for AGE, RS and REPFREQ 
International Journal of Aviation, Aeronautics, and Aerospace, Vol. 3 [2016], Iss. 3, Art. 7

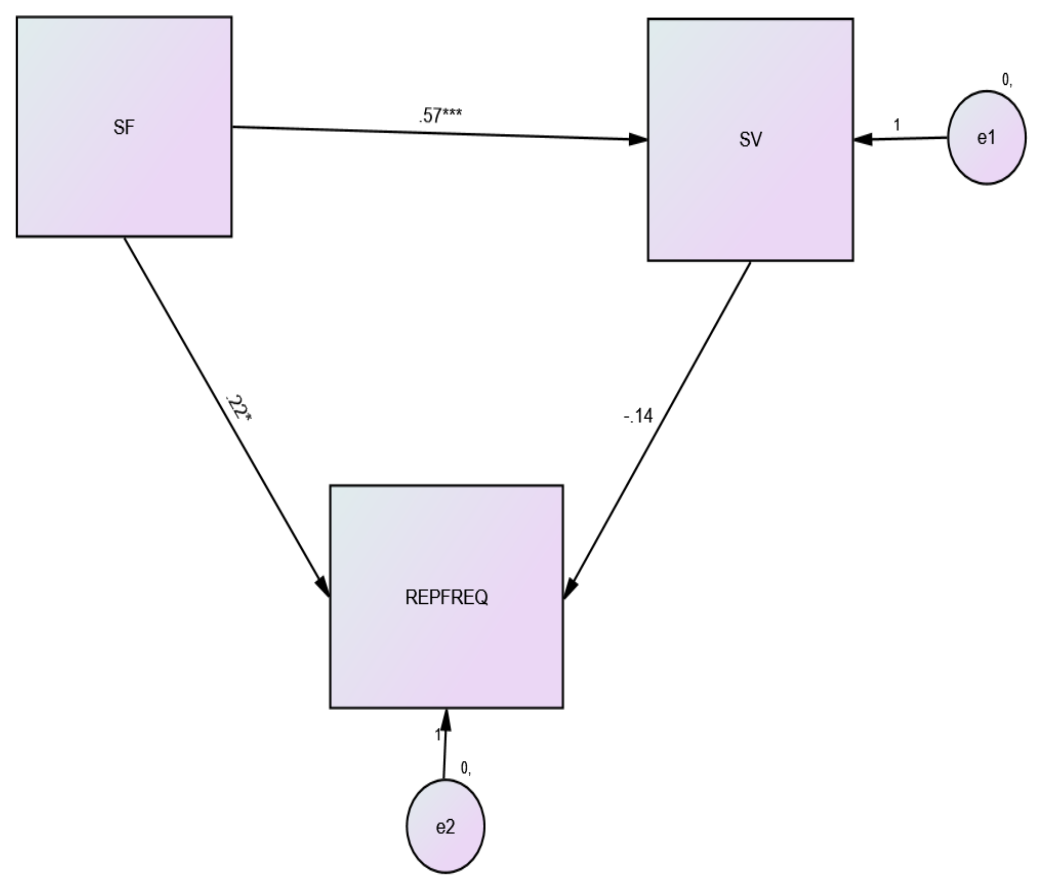

Figure A2. Final Path Model for Mediation Analysis for SF, SV and REPFREQ 
Table A2

Factor Loadings of the Reporting System (RS) Scale

\section{Factor Matrix ${ }^{\mathrm{a}}$}

Factor loadings

\section{Reporting System}

1

RS1_1- The safety reporting system is convenient and easy to use.

RS1_2- Students in my major can report safety discrepancies without fear of negative repercussions.

RS1_3- Students in my major are willing to report information regarding marginal performance or unsafe actions of other students.

RS1_6- I am familiar with the concepts of Safety Management System

RS1_7- I know how and where to report safety related concerns in the aviation department.

Extraction Method: Principal Axis Factoring.

a. 1 factors extracted. 7 iterations required. 
Table A3

Factor Loadings of the Response and Feedback (RF) Scale

Factor Matrix ${ }^{\mathrm{a}}$

Factor

Loadings

1

RF1_1-Safety issues raised by students in my major are communicated regularly to all other students within the major.

RF1_2-When a student in my major reports a safety problem, it is corrected in a timely manner.

RF1_3-Students in my major are satisfied with the way the aviation department/school deals with aviation safety reports.

RF1_5-My aviation department/school keeps confidential database of responses and feedback.

Extraction Method: Principal Axis Factoring.

a. 1 factors extracted. 9 iterations required. 
Table A4

Factor Loadings of the Safety Value (SV) Scale

Factor Matrix ${ }^{a}$

Factor

Loadings

1

\begin{tabular}{lllllllll}
\hline SV1_1 Likert & -Safety & is a core value in my aviation & & .574
\end{tabular} department/school.

SV1_2 Revb - The leadership in my aviation department/school is more concerned with making more money than being safe.

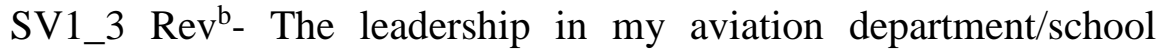
doesn't show much concern for safety until there is an accident or $\quad .828$ incident.

SV1_4 Likert - The leadership in my aviation department/school does not cut corners where safety is concerned.

Extraction Method: Principal Axis Factoring.

a. 1 factors extracted. 9 iterations required.

b. Rev - Reverse Coded 
Table A5

Factor Loadings of the Safety Fundamentals (SF) Scale

Factor Matrix $^{\mathrm{a}}$

Factor Loadings

1

SF1_1Likert -Safety instructions and procedures are easy to $\quad .710$ understand

SF1_2 Likert -My aviation department/school's safety procedures manual are carefully kept up to date.

SF1_3Likert -My aviation department/school is willing to invest money, resources and effort to improve safety.

SF1_4Likert -My aviation department/school is committed to equipping the aviation department with up-to-date technology.

Extraction Method: Principal Axis Factoring.

a. 1 factors extracted. 9 iterations required. 


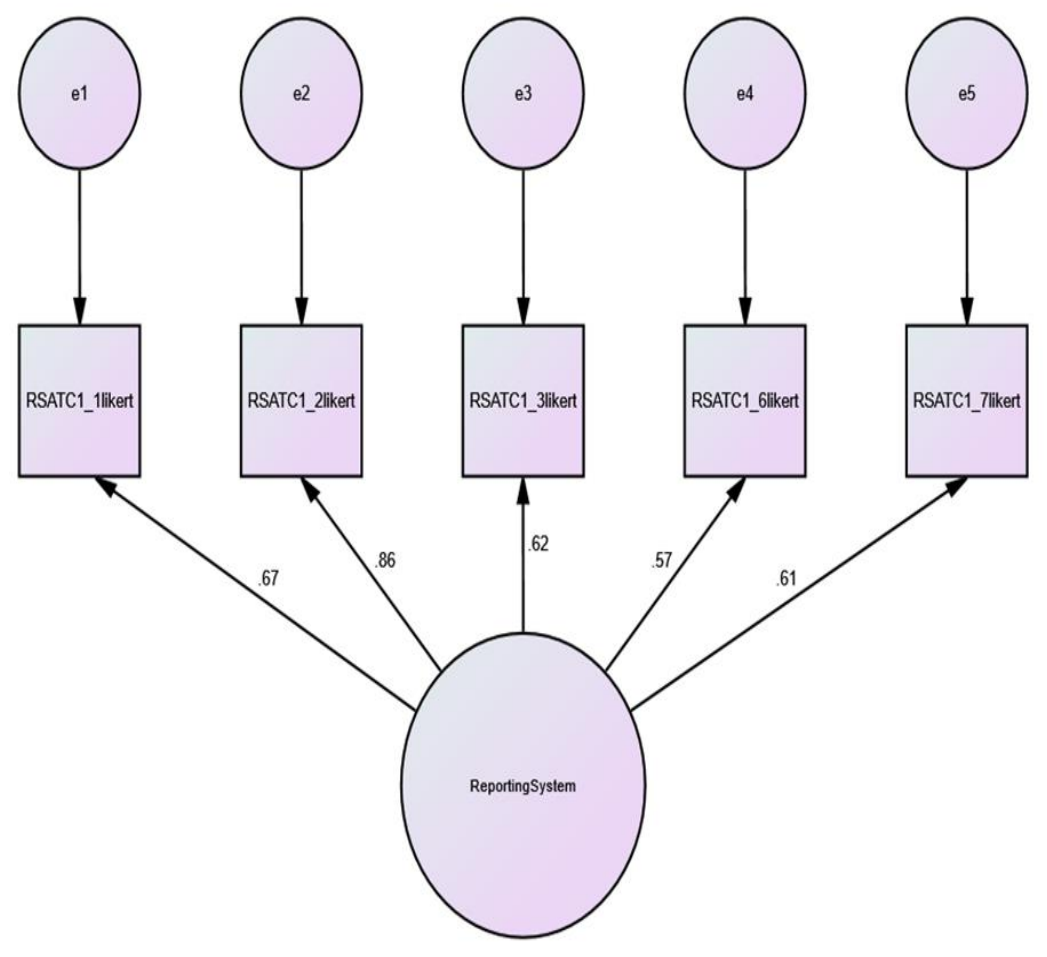

Figure A3. Final Measurement Model (SEM) of Reporting System (RS) 


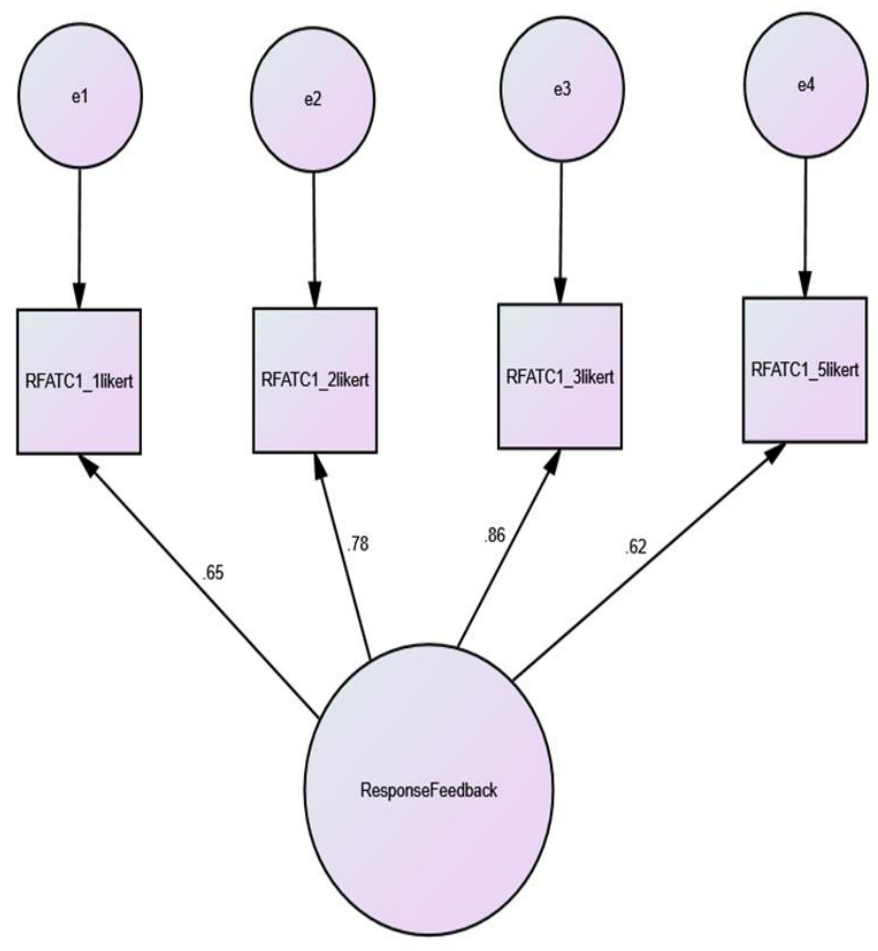

Figure A4. Final Measurement Model (SEM) of Response and Feedback (RF) 


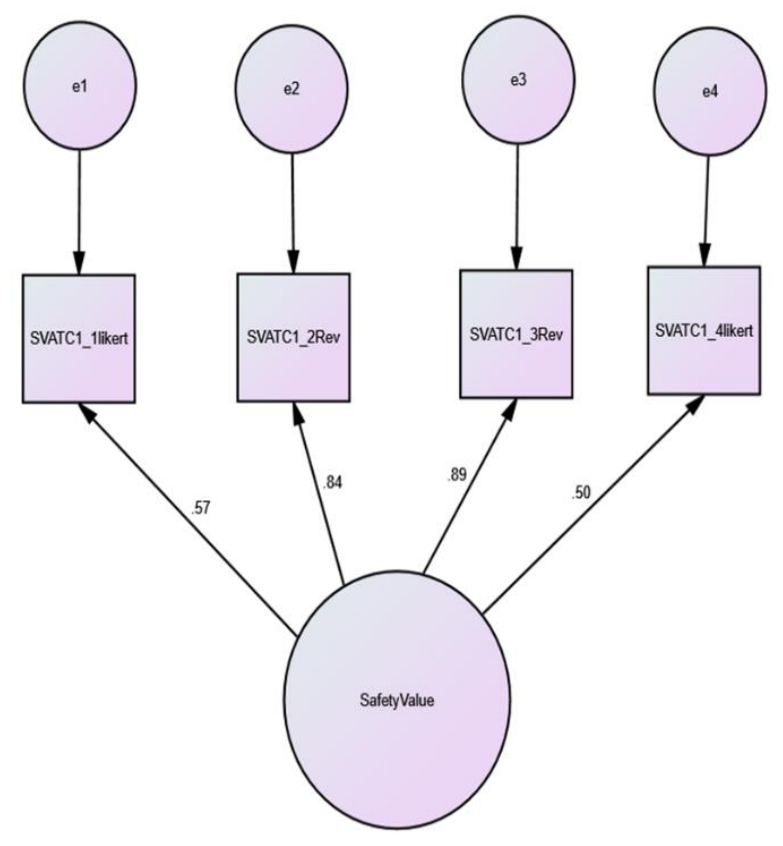

Figure A5. Final Measurement Model (SEM) of Safety Value (SV) 


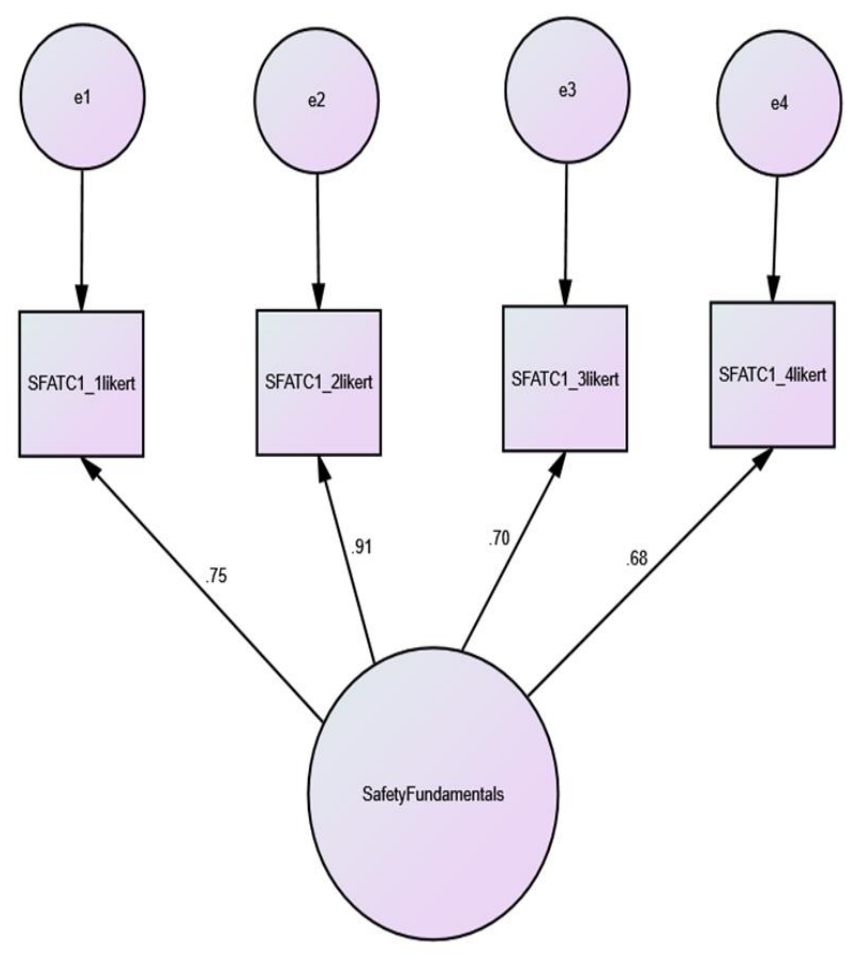

Figure A6. Final Measurement Model (SEM) of Safety Fundamentals (SF) 